\title{
Robustness to Diffusion of Prediction-based Control for Convection Processes
}

\author{
Delphine Bresch-Pietri, Miroslav Krstic
}

\begin{abstract}
In this paper, we consider an Ordinary Differential Equation (ODE) with convection and diffusion in the actuation path. We prove that a prediction-based controller, designed to compensate for the sole convective PDE, actually achieves exponential stabilization of the complete plant, provided that diffusion is small enough. Our result is obtained in $\mathscr{L}_{p}$ norm and covers two cases, full-state feedback and boundary feedback. Simulation results emphasize the validity of this approach.
\end{abstract}

\section{INTRODUCTION}

For linear systems subject to input-delay, prediction-based control strategies, more commonly known as Smith Predictor (see [1],[14],[20],) are state-of-the-art for systems with constant input time-delays (see for instance [2],[4],[9],[11],[15], or [19] and the references therein). Grounding on the use of a prediction of the system state on a time horizon equal to the delay, this technique aims at compensating it, which notably improves transient performances.

When the actuation path is subject to both convection and diffusion, this technique has been recently extended in [12], [21] (see also [8] for an adaptive version) to compensate for both effects. However, while delay dynamics can be finitely stabilized, diffusion compensation should then be understood differently as it introduces an infinite relative degree. Correspondingly, while delay compensation requires to predict future values of the ODE state, in the diffusion case, the control law mainly accounts for an inversion of the diffusion dynamics.

When the diffusive effect can be neglected with respect to the convective one, one may be interested in using a standard prediction-based control law to compensate only for the dominant delay dynamics and, e.g., decrease the controller complexity. However, a well-known fact about predictionbased techniques [18] is that they suffer from being sensitive to various disturbances such as delay mismatch or, to a lesser extent, plant parameters uncertainties [16][17][23]. Thus, there is no guarantee a priori that a prediction-based feedback is robust to diffusion in the actuation path.

For this reason, in this paper, we consider a convection/diffusion PDE-ODE cascade coupled with a predictionbased controller. To analyze the resulting closed-loop robustness, we follow the distributed parameters systems methodology introduced in [13] which has proved to be effective to conclude on the robustness of prediction-based controller

D. Bresch-Pietri (corresponding author) is with CNRS at the Department of Automatic Control, Gipsa-lab, 11 rue des Mathématiques, 38000 Grenoble, France Email: delphine.bresch-pietri@gipsa-lab.fr

M. Krstic is with the Department of Mechanical and Aerospace Engineering, University of California, San Diego, La Jolla CA 92093, USA to several classes of uncertainties [6], [4], [7]. In details, focusing on the sole convection effect in the actuation path, we propose to apply to the distributed input a backstepping transformation which has been developed for input-delay systems. This allows us to map the original system into a target system which is suitable for Lyapunov stability analysis. We investigate two distinct frameworks, full-state feedback and boundary feedback, and show that, in both cases, exponential stabilization is obtained in the sense of the $\mathscr{L}_{p}$ norm, provided that the diffusion parameter is small enough. This is the main contribution.

The paper is organized as follows. In Section II, we start by presenting the problem under consideration. Then, in Section III and IV, we tackle the cases of full-state and boundary control respectively and present our robustness results along with their proofs. This robustness property is illustrated with a numerical example in Section V. We conclude by sketching directions of future work.

\section{PRoblem STATEMENT AND CONTROL DESIGN}

Consider the following ODE-PDE cascade

$$
\left\{\begin{aligned}
\dot{X}(t) & =A X(t)+B u(0, t) \\
u_{t}(x, t) & =u_{x}(x, t)+\varepsilon u_{x x}(x, t) \\
u(1, t) & =U(t) \\
\varepsilon u_{x}(0, t) & =0
\end{aligned}\right.
$$

in which $X \in \mathbb{R}^{n}$, the control $U$ is scalar, $u(\cdot, 0) \in$ $\mathscr{C}\left(\mathbb{R}, \mathscr{H}_{1}([0,1])\right)$ and $\varepsilon \geq 0$ is a small parameter. We consider in the following that the ODE state $X$ is measured and that the pair $(A, B)$ is controllable. In the sequel, we will consider $K$ such that $A+B K$ is Hurwitz.

When $\varepsilon=0$, system (1) is an input-delay system, with a unitary delay. It is well-known that the following control law compensates for the delay

$$
\begin{aligned}
U(t) & =K\left(e^{A} X(t)+\int_{t-1}^{t} e^{A(t-s)} B U(s) d s\right) \\
& =K\left(e^{A} X(t)+\int_{0}^{1} e^{A(1-x)} B u(x, t) d x\right)
\end{aligned}
$$

namely that, after one unit of time, the control law (2) yields the delay-free dynamics $\dot{X}(t)=(A+B K) X(t)$ which is exponentially stable. It is also worth noticing that, when $\varepsilon=0$, one can solve explicitly the transport PDE and obtain $u(x, t)=U(t+x-1)$ which explains the second expression provided in (2).

In this paper, we aim at studying the stability of the closedloop system (1)-(2) in presence of diffusion, that is, for $\varepsilon \geq$ 
0 . As, when $\varepsilon \neq 0$, the two expressions provided in (2) are not equivalent, we consider them separately in the following.

\section{FULL-STATE FEEDBACK}

In this section, we consider that $u(\cdot, t)$ is measured. This corresponds to a dynamical system where convection/diffusion arises from a physical transport process, as opposed to computational or communication-based delay. Consequently, we select the control law as

$$
U(t)=K\left(e^{A} X(t)+\int_{0}^{1} e^{A(1-x)} B u(x, t) d x\right)
$$

We have the following robustness result.

Theorem 1: Consider the closed-loop system consisting of the plant (1) and the control law (3). Define the following functional

$$
\Gamma_{p}(t)=|X(t)|^{2 p}+\int_{0}^{1} u(x, t)^{2 p} d x
$$

for a given $p \in \mathbb{N}^{*}$. There exists $\varepsilon_{p}^{\star}>0$ such that, if $\varepsilon<\varepsilon_{p}^{\star}$, then there exist $\rho>0$ (independent of $p$ ) and $R_{p}>0$ such that

$$
\Gamma_{p}(t) \leq R_{p} \Gamma_{p}(0) e^{-2 p \rho t}, \quad t \geq 0
$$

This result states that the desired exponential convergence is achieved, provided that diffusion is small enough. It is worth noticing that this result holds for any $p \in \mathbb{N}^{*}$, but that the corresponding diffusion coefficient bound $\varepsilon_{p}^{\star}$ decreases with $p$. Thus, one cannot obtain exponential stability in the infinity norm (see Section III-B for more details on this point).

We now provide the proof of this result.

\section{A. Proof of Theorem 1}

1) Backstepping transformation: We consider the following backstepping transformation of the distributed state

$$
w(x, t)=u(x, t)-\int_{0}^{x} K e^{A(x-y)} B u(y, t) d y-K e^{A x} X(t)
$$

Lemma 1: The backstepping transformation (6), along with the control law (3), transform the plant (1) into the following target system

$$
\left\{\begin{aligned}
\dot{X} & =(A+B K) X(t)+B w(0, t) \\
w_{t}(x, t) & =w_{x}(x, t)+\varepsilon w_{x x}(x, t)+\varepsilon g(x, t) \\
w(1, t) & =0 \\
\varepsilon w_{x}(0, t) & =-\varepsilon(K B w(0, t)+K(A+B K) X(t))
\end{aligned}\right.
$$

in which

$$
g(x, t)=K e^{A x} A((A+B K) X(t)+B w(0, t))
$$

Proof: Taking a time-derivative of (6) and using an integration by parts, one obtains

$w_{t}=u_{t}-K B u(x, t)-K \int_{0}^{x} e^{A(x-y)}\left(A B u+\varepsilon B u_{x x}\right) d y-K e^{A x} A X$
Similarly, one obtains for the first and second-order spatial derivatives

$$
\begin{aligned}
w_{x}= & u_{x}-K B u(x, t)-K \int_{0}^{x} A e^{A(x-y)} B u(y, t) d y-K e^{A x} A X(10) \\
w_{x x}= & u_{x x}-K B u_{x}(x, t)-K A B u(x, t)-K \int_{0}^{x} A^{2} e^{A(x-y)} B u(y, t) d y \\
& -K e^{A x} A^{2} X
\end{aligned}
$$

Thus, it follows that

$$
\begin{aligned}
w_{t}= & w_{x}+\varepsilon w_{x x}+\varepsilon\left[-K \int_{0}^{x} e^{A(x-y)} B u_{x x} d y+K B u_{x}(x, t)\right. \\
& \left.+K A B u(x, t)+K \int_{0}^{x} A^{2} e^{A(x-y)} B u(y, t) d y+K e^{A x} A^{2} X\right]
\end{aligned}
$$

which gives the expected result with an integration by parts. The boundary conditions in (7) follow straightforwardly respectively from the definition of the control law (3) and the backstepping transformation (6), and from equation (10) evaluated at $x=0$.

2) Stability study in $\mathscr{L}_{p}$-norm: Define the following Lyapunov functional candidate

$$
V_{p}(t)=\left(X^{T} P X\right)^{p}+b \int_{0}^{1} e^{2 p x} w(x, t)^{2 p} d x
$$

in which $b$ is a positive constant, $p \in \mathbb{N}^{*}$ and $P$ is the solution to the Lyapunov equation associated to $A+B K$ and a given positive definite matrix $Q$. For the sake of conciseness, in the sequel, we denote $\|w(t)\|_{2 p}^{2 p}=\int_{0}^{1} e^{2 p x} w(x, t)^{2 p} d x$.

Taking a time-derivative of (13), one gets

$$
\begin{gathered}
\dot{V}_{p}(t)=-p\left(X^{T} P X\right)^{p-1} X^{T} Q X+2 p\left(X^{T} P X\right)^{p-1} X^{T} P B w(0, t) \\
-b w(0, t)^{2 p}-2 p b\|w(t)\|_{2 p}^{2 p}+2 p b \varepsilon \int_{0}^{1} e^{2 p x} w^{2 p-1} w_{x x} d x \\
+2 p b \varepsilon \int_{0}^{1} e^{2 p x} g(x, t) w^{2 p-1}(x, t) d x
\end{gathered}
$$

Performing an integration by parts, one obtains

$$
\begin{aligned}
\dot{V}_{p}(t) & =-p\left(X^{T} P X\right)^{p-1} X^{T} Q X+2 p\left(X^{T} P X\right)^{p-1} X^{T} P B w(0, t) \\
& -b w(0, t)^{2 p}-2 p b\|w(t)\|_{2 p}^{2 p}+\varepsilon b\left(2 p w(0, t)^{2 p}\right. \\
& -2 p w(0, t)^{2 p-1} w_{x}(0, t)+(2 p)^{2}\|w(t)\|_{2 p}^{2 p} \\
& \left.-\int_{0}^{1} 2 p(2 p-1) e^{2 p x} w(x, t)^{2 p-2} w_{x}(x, t)^{2} d x\right) \\
& +2 p b \varepsilon \int_{0}^{1} e^{2 p x} g(x, t) w(x, t)^{2 p-1} d x
\end{aligned}
$$

Further, with an integration by parts and applying Young inequality, one can observe that

$$
\begin{aligned}
& 2 p \int_{0}^{1} e^{2 p x} w(x, t)^{2 p} d x \\
& =-w(0, t)^{2 p}-2 p \int_{0}^{1} e^{2 p x} w(x, t)^{2 p-1} w_{x}(x, t) d x \\
& \leq-w(0, t)^{2 p}+p \int_{0}^{1} e^{2 p x} w(x, t)^{2 p-2} w_{x}(x, t)^{2} d x \\
& \quad+p \int_{0}^{1} e^{2 p x} w(x, t)^{2 p} d x
\end{aligned}
$$


and thus that

$$
\begin{array}{rl}
-p \int_{0}^{1} e^{2 p x} w(x, t)^{2 p-2} w_{x}(x, t)^{2} & d x \\
\leq-w(0, t)^{2 p}-p\|w(t)\|_{2 p}^{2 p}
\end{array}
$$

Consequently, one gets

$$
\begin{aligned}
\dot{V}_{p}(t) \leq & -p \underline{\lambda}(Q)\left(X^{T} P X\right)^{p-1}|X|^{2}+2 p\left(X^{T} P X\right)^{p-1}|X||P B w(0, t)| \\
& -b w(0, t)^{2 p}-2 p b\|w(t)\|_{2 p}^{2 p}+\varepsilon b\left(2 p w(0, t)^{2 p}\right. \\
& -2 p w(0, t)^{2 p-1} w_{x}(0, t)-2(2 p-1) w(0, t)^{2 p} \\
& \left.-2 p(2 p-1)\|w(t)\|_{2 p}^{2 p}+(2 p)^{2}\|w(t)\|_{2 p}^{2 p}\right) \\
& +2 p b \varepsilon \int_{0}^{1} e^{2 p x} g(x, t) w(x, t)^{2 p-1} d x
\end{aligned}
$$

From the definition of the function $g$ in (8) and applying Young inequality, one obtains the existence of a positive constant $M>0$ (which does not depend on $p$ ) such that

$$
\begin{aligned}
& 2 p \int_{0}^{1} e^{2 p x} g(x, t) w(x, t)^{2 p-1} d x \\
& \quad \leq M\left(2 p\|w(t)\|_{2 p}^{2 p}+w(0, t)^{2 p}+|X(t)|^{2 p}\right)
\end{aligned}
$$

Similarly, one gets

$$
\begin{array}{rl}
2 & p\left(X^{T} P X\right)^{p-1}|X||P B w(0, t)| \\
\leq & (2 p-1) \frac{\underline{\lambda}(Q)}{4} \frac{\lambda}{\bar{\lambda}(P)}\left(\left(X^{T} P X\right)^{p-1}|X(t)|\right)^{\frac{2 p}{2 p-1}} \\
& +\left(\frac{4}{\underline{\lambda}(Q)} \frac{\bar{\lambda}(P)}{\underline{\lambda}(P)}\right)^{2 p-1}|P B|^{2 p} w(0, t)^{2 p} \\
\leq & \frac{p \underline{\lambda}(Q)}{2}\left(X^{T} P X\right)^{p-1}|X(t)|^{2} \\
& +\left(\frac{4}{\underline{\lambda}(Q)} \underline{\underline{\lambda}(P)}\right)^{2 p-1}|P B|^{2 p} w(0, t)^{2 p}
\end{array}
$$

in which $\underline{\lambda}(\cdot)$ and $\bar{\lambda}(\cdot)$ denote respectively the minimum and maximum eigenvalues of a given matrix, and

$$
\begin{aligned}
-2 p w(0, t)^{2 p-1} w_{x}(0, t) \leq & 2 p K B w(0, t)^{2 p}+(2 p-1) w(0, t)^{2 p} \\
& +|K(A+B K)|^{2 p}|X(t)|^{2 p}
\end{aligned}
$$

Plugging these inequalities into (18), one gets

$$
\begin{aligned}
\dot{V}_{p}(t) & \leq-\frac{p \underline{\lambda}(Q)}{4}\left(X^{T} P X\right)^{p-1}|X|^{2} \\
& -\left(\frac{p \underline{\lambda}(Q) \underline{\lambda}(P)^{p-1}}{4}-b \varepsilon\left(M+|K(A+B K)|^{2 p}\right)\right)|X|^{2 p} \\
& -2 p b(1-\varepsilon(M+1))\|w(t)\|_{2 p}^{2 p} \\
& -\left(b-\left(\frac{4}{\bar{\lambda}(Q)} \frac{\bar{\lambda}(P)}{\bar{\lambda}(P)}\right)^{2 p-1}|P B|^{2 p}\right. \\
& -b \varepsilon(1+M+2 p|K B|)) w(0, t)^{2 p}
\end{aligned}
$$

Assume that

$$
b>2\left(\frac{4 \bar{\lambda}(P)}{\underline{\lambda}(Q) \underline{\lambda}(P)}\right)^{2 p-1}|P B|^{2 p}
$$

and that $\varepsilon<\varepsilon_{p}^{\star}$ with

$$
\varepsilon_{p}^{\star}=\min \left\{\frac{p \underline{\lambda}(Q) \underline{\lambda}(P)^{p-1}}{4 b\left(M+|K(A+B K)|^{2 p}\right)}, \frac{1}{2(M+1+2 p|K B|)}\right\}
$$

then, one obtains

$$
\dot{V}_{p}(t) \leq-2 p \min \left\{\frac{\underline{\lambda}(Q)}{8 \bar{\lambda}(P)}, \frac{1}{2}\right\} V_{p}(t)
$$

which gives

$$
V_{p}(t) \leq e^{-2 p \min \left\{\frac{\lambda(Q)}{8 \bar{\lambda}(P)}, \frac{1}{2}\right\}^{t}} V_{p}(0)
$$

3) Equivalence between $V_{p}$ and $\Gamma_{p}$ : To conclude the proof, it now remains to show that $V_{p}$ and $\Gamma_{p}$ are equivalent, i.e., that there exist $c_{1}, c_{2}>0$ such that $c_{1} V_{p} \leq \Gamma_{p} \leq c_{2} V_{p}$. This fact actually follows straightforwardly by applying Young inequality to (6) and its inverse

$u(x, t)=w(x, t)+\int_{0}^{x} K e^{(A+B K)(x-y)} B w(y, t) d y+K e^{(A+B K) x} X(t)$

However, note that these constants $c_{1}$ and $c_{2}$ depend on $p$. Then, it follows from (26) that (5) holds with $R_{p}=\frac{c_{2}}{c_{1}}$ and $\rho=\min \left\{\frac{\lambda(Q)}{8 \bar{\lambda}(P)}, \frac{1}{2}\right\}$.

\section{$B$. Comments on the norm selection}

It is worth noting that, even if our robustness result holds for all $\mathscr{L}_{p}$ norm, it does not extend to the infinity norm. Indeed, the bound $\varepsilon_{p}^{\star}$ defined in (24) depends on the value of $p$ and tends to zero as $p$ tends to infinity. Thus, according to our analysis at least, prediction-based control is not robust to diffusion in infinity norm. Interestingly, the same conclusion can be obtained by studying the system via Input-to-State Stability and attempting to apply the Small Gain Theorem.

\section{BOUNDARY FEEDBACK}

In this section, as the distributed input is seldom measured in application, we consider the more realistic case in which the actuator state is unknown. Consequently, we choose the control law as

$$
U(t)=K\left[e^{A} X(t)+\int_{t-1}^{t} e^{A(t-s)} B U(s) d s\right]
$$

In this case, a similar result to the one provided in the previous section holds.

Theorem 2: Consider the closed-loop system consisting of the plant (1) and the control law (28). Define the following functional

$$
\begin{aligned}
\Upsilon_{p}(t)= & |X(t)|^{2 p}+\int_{t-1}^{t} U(s)^{2 p} d s \\
& +\int_{t-1}^{t} \dot{U}(s)^{2 p} d s+\int_{t-1}^{t} \ddot{U}(s)^{2 p} d s+\int_{0}^{1} u(x, t)^{2 p} d x
\end{aligned}
$$


for a given $p \in \mathbb{N}$. For any initial condition $U_{0}: s \in[-1,0] \mapsto$ $U(s) \in \mathscr{C}^{2}([-1,0], \mathbb{R})$, there exists $\varepsilon_{p}^{\star}>0$ such that, if $\varepsilon<\varepsilon_{p}^{\star}$, then there exist $\rho>0$ (independent of $p$ ) and $R_{p}>0$ such that

$$
\Upsilon_{p}(t) \leq R_{p} \Upsilon_{p}(0) e^{-2 p \rho t}, \quad t \geq 0
$$

The definition (29) of the functional $\Upsilon$ is due to the specific form of Lyapunov functional considered in the following proof.

\section{Proof of Theorem 2:}

To account for the fact that the control law explicitly neglects diffusion in the actuator path, we define the distributed estimate $\hat{u}(x, t)=U(t+x-1), x \in[0,1]$. Thus, the controller (28) can be reformulated as

$$
U(t)=K\left[e^{A} X(t)+\int_{0}^{1} e^{A(1-x)} B \hat{u}(x, t) d x\right]
$$

and, introducing the estimation error $\tilde{u}=u-\hat{u}$, one obtains

$$
\left\{\begin{aligned}
\dot{X}(t) & =A X(t)+B[\tilde{u}(0, t)+\hat{u}(0, t)] \\
\tilde{u}_{t}(x, t) & =\tilde{u}_{x}(x, t)+\varepsilon\left(\tilde{u}_{x x}(x, t)+\hat{u}_{x x}(x, t)\right) \\
\tilde{u}(1, t) & =0 \\
\varepsilon \tilde{u}_{x}(0, t) & =-\varepsilon \hat{u}_{x}(0, t) \\
\hat{u}_{t}(x, t) & =\hat{u}_{x}(x, t) \\
\hat{u}(1, t) & =U(t)
\end{aligned}\right.
$$

We now consider the following backstepping transformation

$$
\hat{w}(x, t)=\hat{u}(x, t)-\int_{0}^{x} K e^{A(x-y)} B \hat{u}(y, t) d y-K e^{A x} X(t)
$$

Following the same steps as in the proof of Lemma 1, one can prove that this transformation, along with the control law (31), map the previous system into

$$
\left\{\begin{aligned}
\dot{X}(t) & =(A+B K) X(t)+B[\tilde{u}(0, t)+\hat{w}(0, t)] \\
\tilde{u}_{t}(x, t) & =\tilde{u}_{x}(x, t)+\varepsilon\left(\tilde{u}_{x x}(x, t)+h(x, t)\right) \\
\tilde{u}(1, t) & =0 \\
\varepsilon \tilde{u}_{x}(0, t) & =-\varepsilon\left[\hat{w}_{x}(0, t)+K B \hat{w}(0, t)+K(A+B K) X(t)\right] \\
\hat{w}_{t}(x, t) & =\hat{w}_{x}(x, t) \\
\hat{w}(1, t) & =0
\end{aligned}\right.
$$

in which, considering the inverse backstepping transformation (similar to the one defined in the previous section in (27))

$$
\begin{aligned}
h(x, t)= & \hat{w}_{x x}(x, t)+K B \hat{w}_{x}(x, t)+K(A+B K) B \hat{w}(x, t) \\
& +K \int_{0}^{x}(A+B K)^{2} e^{(A+B K)(x-y)} B \hat{w}(y, t) d y \\
& +K e^{(A+B K) x}(A+B K)^{2} X(t)
\end{aligned}
$$

In the following analysis, we also need the dynamics of the first and second spatial-derivatives of the backstepping variable which is simply

$$
\left\{\begin{array} { l } 
{ \hat { w } _ { x t } ( x , t ) = \hat { w } _ { x x } ( x , t ) } \\
{ \hat { w } _ { x } ( 1 , t ) = 0 }
\end{array} \quad \left\{\begin{array}{l}
\hat{w}_{x x t}(x, t)=\hat{w}_{x x x}(x, t) \\
\hat{w}_{x x}(1, t)=0
\end{array}\right.\right.
$$

We are now ready to start the Lyapunov analysis. We consider the following functional candidate

$$
\begin{aligned}
V_{p}(t)= & \left(X^{T} P X\right)^{p}+b \int_{0}^{1} e^{2 p x} \tilde{u}(x, t)^{2 p} d x \\
& +b \int_{0}^{1} e^{2 p x}\left(\hat{w}(x, t)^{2 p}+\hat{w}_{x}(x, t)^{2 p}+\hat{w}_{x x}(x, t)^{2 p}\right) d x
\end{aligned}
$$

in which $b>0, p \in \mathbb{N}^{*}$ and $P$ is the solution to the Lyapunov equation associated to $A+B K$ and a given positive definite matrix $Q$. Taking a time-derivative and performing integrations by parts, one obtains

$$
\begin{aligned}
\dot{V}_{p}(t) & =-p\left(X^{T} P X\right)^{p-1} X^{T} Q X-b \tilde{u}(0, t)^{2 p}-2 p b\|\tilde{u}(t)\|_{2 p}^{2 p} \\
& +2 p\left(X^{T} P X\right)^{p-1} X^{T} P B[\tilde{u}(0, t)+\hat{w}(0, t)] \\
& -b\left(\hat{w}(0, t)^{2 p}+\hat{w}_{x}(0, t)^{2 p}+\hat{w}_{x x}(0, t)^{2 p}\right) \\
& -2 p b\left(\|\hat{w}(t)\|_{2 p}^{2 p}+\left\|\hat{w}_{x}(t)\right\|_{2 p}^{2 p}+\left\|\hat{w}_{x x}(t)\right\|_{2 p}^{2 p}\right) \\
& +2 p b \varepsilon \int_{0}^{1} e^{2 p x} \tilde{u}(x, t)^{2 p-1}\left(\tilde{u}_{x x}+h(x, t)\right) d x
\end{aligned}
$$

in which, using integrations by parts,

$$
\begin{aligned}
& \int_{0}^{1} e^{2 p x} \tilde{u}(x, t)^{2 p-1} \tilde{u}_{x x} d x \\
& =-\tilde{u}(0, t)^{2 p-1} \tilde{u}_{x}(0, t)+\tilde{u}(0, t)^{2 p}+2 p\|\tilde{u}(t)\|_{2 p}^{2 p} \\
& \quad-(2 p-1) \int_{0}^{1} e^{2 p x} \tilde{u}^{2 p-2} \tilde{u}_{x}^{2} d x
\end{aligned}
$$

Following similar steps as in (16)-(17), one gets

$$
\begin{aligned}
2 p \int_{0}^{1} e^{2 p x} \tilde{u}(x, t)^{2 p-1} \tilde{u}_{x x} d x & \\
\leq & -2 p \tilde{u}(0, t)^{2 p-1} \tilde{u}_{x}(0, t)+\tilde{u}(0, t)^{2 p}+2 p\|\tilde{u}(t)\|_{2 p}^{2 p} \\
\leq & (3+|K B|) \tilde{u}(0, t)^{2 p}+\hat{w}_{x}(0, t)^{2 p}+|K B| \hat{w}(0, t)^{2 p} \\
& \quad+2 p\|\tilde{u}(t)\|_{2 p}^{2 p}+|K(A+B K)|^{2 p}|X|^{2 p}
\end{aligned}
$$

in which the last inequality is obtained using the expression of $\tilde{u}_{x}(0, t)$ in (34) and Young inequality. Further, from (35), applying Young inequality and integrations by parts, one obtains the existence of a constant $\tilde{M}>0$ (independent of p) such that

$$
\begin{aligned}
& 2 p \int_{0}^{1} e^{2 p x} \tilde{u}(x, t)^{2 p-1} h(x, t) d x \leq \tilde{M}\left(2 p\|\tilde{u}(t)\|_{2 p}^{2 p}\right. \\
& \left.\quad+\|\hat{w}(t)\|_{2 p}^{2 p}+\left\|\hat{w}_{x}(t)\right\|_{2 p}^{2 p}+\left\|\hat{w}_{x x}(t)\right\|_{2 p}^{2 p}+|X|^{2 p}\right)
\end{aligned}
$$

Plugging together (38), (40) and (41), one obtains, using Young inequality,

$$
\begin{aligned}
\dot{V}_{p}(t) & \leq-p \frac{\underline{\lambda}(Q)}{4}\left(X^{T} P X\right)^{p-1}|X|^{2} \\
& -\left(b-\left(\frac{8 \bar{\lambda}(P)}{\underline{\lambda}(Q) \underline{\lambda}(P)}\right)|P B|^{2 p}-b \varepsilon|K B|\right) \hat{w}(0, t)^{2 p} \\
& -\left(p \frac{\underline{\lambda}(Q) \underline{\lambda}(P)^{p-1}}{4}-\varepsilon b\left(\tilde{M}+|K(A+B K)|^{2 p}\right)\right)|X(t)|^{2 p} \\
& -\left(b-\left(\frac{8 \bar{\lambda}(P)}{\underline{\lambda}(Q) \underline{\lambda}(P)}\right)^{2 p-1}|P B|^{2 p}-b \varepsilon(3+|K B|)\right) \tilde{u}(0, t)^{2 p}
\end{aligned}
$$




$$
\begin{aligned}
& -b(1-\varepsilon) \hat{w}_{x}(0, t)^{2}-2 p b(1-\varepsilon(1+\tilde{M}))\|\tilde{u}(t)\|_{2 p}^{2 p} \\
& -2 p b(1-\varepsilon)\left(\|\hat{w}(t)\|_{2 p}^{2 p}+\left\|\hat{w}_{x}(t)\right\|_{2 p}^{2 p}+\left\|\hat{w}_{x x}(t)\right\|_{2 p}^{2 p}\right)
\end{aligned}
$$

Consequently, choosing

$$
b>2\left(\frac{8 \bar{\lambda}(P)}{\underline{\lambda}(Q) \underline{\lambda}(P)}\right)^{2 p-1}|P B|^{2 p}
$$

and if $\varepsilon<\varepsilon_{p}^{\star}$ with

$$
\varepsilon_{p}^{\star}=\min \left\{\frac{1}{2(3+|K B|)}, \frac{p \underline{\lambda}(Q) \underline{\lambda}(P)^{p-1}}{4 b\left(\tilde{M}+|K(A+B K)|^{2 p}\right)}\right\}
$$

one obtains that

$$
\dot{V}_{p}(t) \leq-2 p \min \left\{\frac{\underline{\lambda}(Q)}{8 \overline{\bar{\lambda}}(P)}, \frac{1}{2}\right\} V_{p}(t)
$$

It now remains to show that $\Upsilon_{p}$ and $V_{p}$ are equivalent. This can be done as previously by considering the inverse backstepping transformation and applying Young inequality. It is also necessary to notice that $\hat{u}_{x}(x, t)=\dot{U}(t+x-1)$ and $\hat{u}_{x x}(x, t)=\ddot{U}(t+x-1)$ which explains the presence of integrals of $\dot{U}$ and $\ddot{U}$ in (29). This concludes the proof.

The same comments as in the previous section regarding the infinity norm apply here.

\section{NUMERICAL SIMULATIONS}

To illustrate the merits of the proposed controller, we consider a numerical example inspired from the fluid flow system considered, e.g., in [5] [10]. It consists in an horizontal tube equipped with inlet and outlet fans and a mist injector. The mist injection is fixed and the control objective is to regulate the moisture at the output of the tube, that is, the ODE state $X$ is the moisture while the control $U$ is the fan power. A schematic view of the set-up is given in Fig. 1.

As studied in [5] [10], a stable first-order delay equation encapsulates the main features of the dynamics under consideration. A more detailed modeling enables to conclude that diffusion also occurs inside the tube, but is negligible compared to convection. The moisture measured at the output thus satisfies (1) with $A=-1 / \tau_{0}$ and $B=K_{P} / \tau_{0}$ and $A+B K$ is Hurwitz for any constant $K<0$. We chose $K=-10$ in simulation.

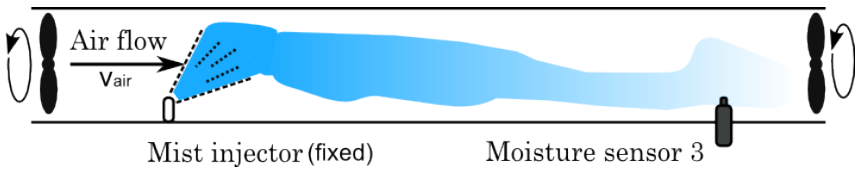

Fig. 1. Schematic view of the flowtube system under consideration as an illustrative example.
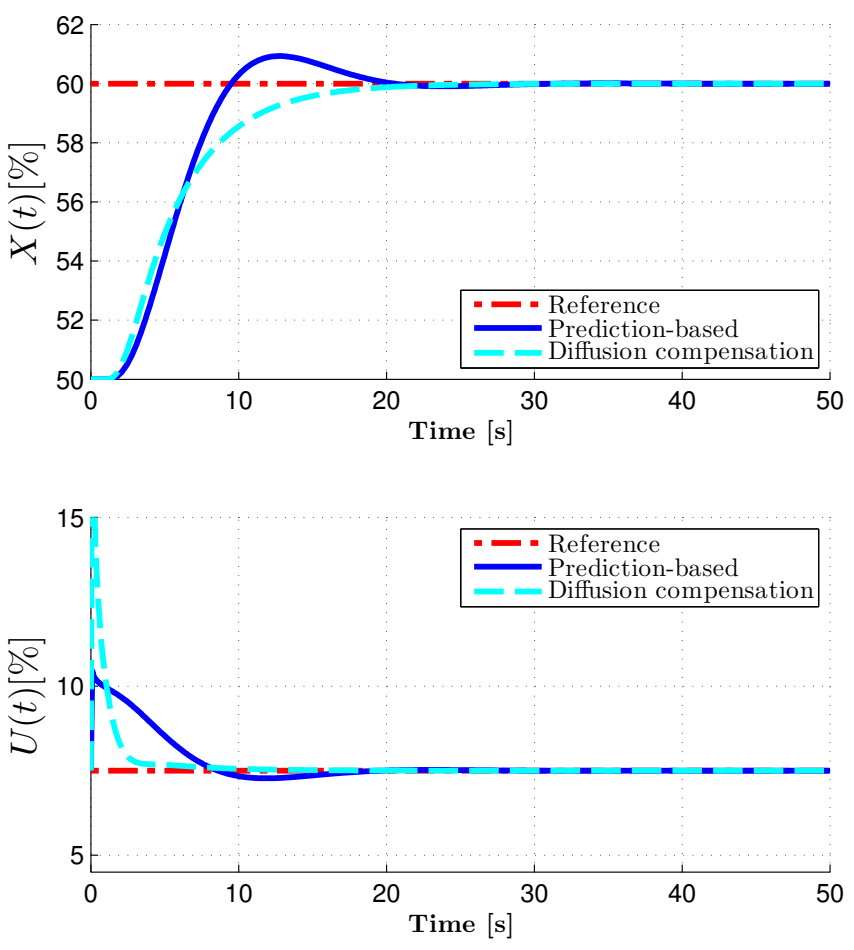

(a) Evolution of the moisture, the fan power and the parameter estimate.
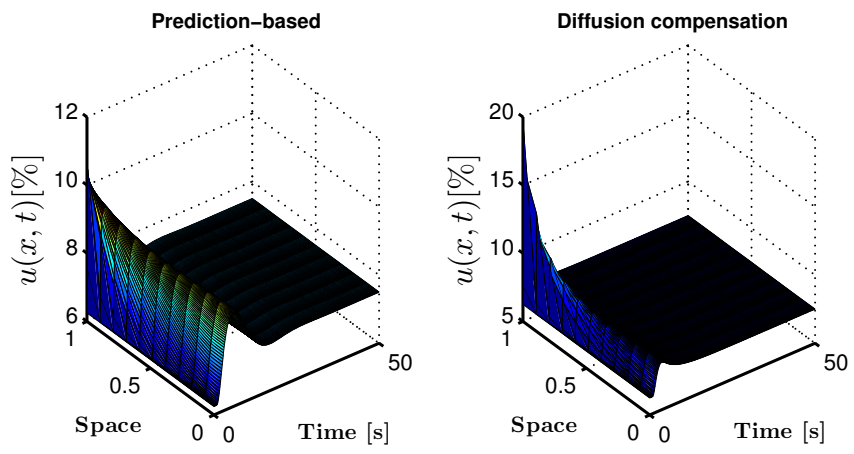

(b) Distributed input.

Fig. 2. Numerical results for the closed-loop dynamics of the moisture modeled by (1) with $A=-1 / \tau_{0}$ and $B(\theta)=K_{P} / \tau_{0}$, for two different controllers. The initial condition corresponds to a moisture (relative humidity) equilibrium of $50 \%$ that one wants to regulate to a new operating condition corresponding to $60 \%$ of moisture. The prediction-based controller (28) has been implemented with a feedback gain $K=-10$. The nominal diffusion/convection controller from [12], [21] has been implemented with a similar feedback gain $K=-10$.

One can easily observe ${ }^{1}$ that the results presented in this paper straightforwardly extend to the case of a non-unitary delay $D$. Thus, for simulation, we take ${ }^{2} D=6 \mathrm{~s}, \tau_{0}=9 \mathrm{~s}$, $\varepsilon=0.04 \mathrm{~s}^{-1}$. This choice leads to a Peclet number of $\varepsilon D=$ 0.24 . As only boundary measurements are available in this example, we implement the controller (28).

We compare the obtained closed-loop performances with the full-state diffusion/convection compensation solution proposed in [12], [21] as a reference. Corresponding results are

\footnotetext{
${ }^{1}$ Performing, from an unnormalized process $D v_{t}=v_{x}+a v_{x x}$, a simple change of variable $u(x, t)=v(x, D t)$

${ }^{2}$ Note that the convection speed is actually time-varying, but we neglect those variations here for the sake of simplicity
} 
depicted in Fig. 2. First, one can observe that convergence is indeed obtained, even if the Peclet number is quite high in this example. Also, one can notice that, when using the proposed prediction-based controller, transient performances are slightly degraded as an overshoot is generated. However, overall, this controller reasonably performs compared to a strategy tailored to handle diffusion and using the distributed state knowledge.

\section{CONCLUSION}

In this paper, we proved that prediction-based controller for linear systems subject to a single input-delay are robust to diffusion in the actuation path. Future works should investigate the extension of this property to systems subject to multiple input-delays [3], [22].

\section{REFERENCES}

[1] Z. Artstein. Linear systems with delayed controls: a reduction. IEEE Transactions on Automatic Control, 27(4):869-879, 1982.

[2] N. Bekiaris-Liberis and M. Krstic. Nonlinear Control Under Nonconstant Delays, volume 25. Society for Industrial and Applied Mathematics, 2013.

[3] N. Bekiaris-Liberis and M. Krstic. Predictor-feedback stabilization of multi-input nonlinear systems. arXiv preprint arXiv:1508.05756, 2015.

[4] D. Bresch-Pietri, J. Chauvin, and N. Petit. Adaptive control scheme for uncertain time-delay systems. Automatica, 48:1536-1552, 2012.

[5] D. Bresch-Pietri and K. Coulon. Prediction-based control of moisture in a convective flow. In Proc. of the European Control Conference, pages 43-48, 2015.

[6] D. Bresch-Pietri and M. Krstic. Adaptive trajectory tracking despite unknown input delay and plant parameters. Automatica, 45(9):20742081, 2009.

[7] D. Bresch-Pietri and M. Krstic. Delay-adaptive control for nonlinear systems. IEEE Transactions on Automatic Control, 59:1203-1218, 2014.

[8] D. Bresch-Pietri and M. Krstic. Adaptive compensation of diffusionadvection actuator dynamics using boundary measurements. In Conference on Decision and Control, 2015.

[9] K. Gu and S. I. Niculescu. Survey on recent results in the stability and control of time-delay systems. Journal of Dynamic Systems, Measurement, and Control, 125:158, 2003.

[10] M. A. Hernandez Perez, E. Witrant, and O. Sename. A time-delay approach for modeling and control of mist in a poiseuille flow. In Proc. of the European Control Conference, 2014.

[11] M. Jankovic. Recursive predictor design for linear systems with time delay. In American Control Conference, pages 4904-4909, 2008.

[12] M. Krstic. Compensating actuator and sensor dynamics governed by diffusion PDEs. Systems and Control Letters, 58:372-377, 2009.

[13] M. Krstic and A. Smyshlyaev. Boundary Control of PDEs: a Course on Backstepping Designs. Society for Industrial and Applied Mathematics Philadelphia, PA, USA, 2008.

[14] A. Manitius and A. Olbrot. Finite spectrum assignment problem for systems with delays. IEEE Transactions on Automatic Control, 24(4):541-552, 1979.

[15] W. Michiels and S. I. Niculescu. Stability and Stabilization of TimeDelay Systems. Society for Industrial and Applied Mathematics, 2007.

[16] S. Mondié, S. Niculescu, and J. J. Loiseau. Delay robustness of closed loop finite assignment for input delay systems. In Proc. of the 3rd IFAC Conference on Time Delay Systems, Santa Fe, New Mexico, USA, 2001.

[17] S. I. Niculescu. Delay Effects on Stability: A Robust Control Approach, volume 269 of Lecture notes in control and information sciences. Springer Verlag, 2001.

[18] Z. Palmor. Stability properties of Smith dead-time compensator controllers. International Journal of Control, 32:937-49, 1980.

[19] J.-P. Richard. Time-delay systems: an overview of some recent advances and open problems. Automatica, 39(10):1667-1694, 2003.

[20] O. J. M. Smith. A controller to overcome dead time. ISA Journal, 6(2):28-33, 1959.
[21] G. A. Susto and M. Krstic. Control of PDE-ODE cascades with neumann interconnections. Journal of the Franklin Institute, 347(1):284314, 2010.

[22] D. Tsubakino, T. Roux Oliveira, and M. Krstic. Predictor-feedback for multi-input lti systems with distinct delays. In American Control Conference (ACC), 2015, pages 571-576. IEEE, 2015.

[23] Q. C. Zhong. Robust Control of Time-Delay Systems. Springer Verlag, 2006. 